Bull. Korean Math. Soc. 47 (2010), No. 3, pp. 513-526

DOI 10.4134/BKMS.2010.47.3.513

\title{
UNIVERSAL HYPERDYNAMICAL SYSTEMS
}

\author{
A. Dehghan Nezhad and B. Davvaz
}

\begin{abstract}
In this paper, the theory of $n$-ary hypergroups and some applications of hyperalgebras (Fredholm-Voltra integral, copula) are studied. We define some new concepts of topological hyperdynamical systems, universal hyperdynamical systems and immersed universal hyperalgebra. Also, we present some results in this respect.
\end{abstract}

\section{Introduction}

Hyperstructure theory was born in 1934 when Marty defined hypergroups as a generalization of groups. This theory has been studied in the following decades and nowadays by many mathematicians. Hypergroup theory both extends some well-known group results and introduced new topics leading thus to a wide variety of applications, as well as to a broadening of the investigation fields. There are applications to the following subjects: geometry, hypergraphs, binary relations, lattices, fuzzy sets and rough sets, automata, cryptography, combinatorics, codes, artificial intelligence, and probabilistic. A comprehensive review of the theory of hyperstructures appears in [3, 4, 20].

$n$-ary generalizations of algebraic structures is the most natural way for further development and deeper understanding of their fundamental properties. Ameri and Zahedi in [2], Davvaz in [6] and Pelea and Purdea in [17] studied algebraic hypersystems. The notion of $n$-hypergroup was first introduced by Davvaz and Vougiouklis as a generalization of $n$-ary group [7], and studied mainly by Leoreanu-Fotea and Davvaz $[12,13,14]$. Leoreanu-Fotea and Davvaz introduced and studied the notion of a partial $n$-hypergroupoid, associated with a binary relation. Some important results, concerning Rosenberg partial hypergroupoids, induced by relations, are generalized to the case of $n$-hypergroupoids

In [11], Ilookashooly and Molaei studied the notion of immersed hypergroup and they considered hypergroups which are also smooth manifolds and their join operators create immersed submanifolds.

Received November 24, 2008.

2000 Mathematics Subject Classification. 20N20.

Key words and phrases. algebra, hyperalgebra, $n$-ary hypergroup, copula, topological space, hyperdynamical system. 
In [8], Hošková generalized the concept of topological groupoid to topological hypergroupoid. Some results of this type can be found in [1].

\section{Basic definitions}

In this section, we give a brief introduction of universal hyperalgebras, $n$ ary hypergroups and homomorphisms between them. First, we recall some notation of ordinary universal algebras.

Definition 2.1. Let $n_{t}$ be a non-negative integer and $H$ be a non-empty set. We denote by $H^{n_{t}}$ the Cartesian product $H \times \cdots \times H$, where $H$ appears $n_{t}$ times. An element of $H^{n}$ will be denoted by $\left(x_{1}, \ldots, x_{n}\right)$, where $x_{i} \in H$ for any $i$ with $1 \leq i \leq n$. An $n_{t}$-ary operation on $H$ is a function $\lambda_{t}$ from $H^{n_{t}}$ to $H$, where $H^{n_{t}}$ is the set of $n_{t}$-tuples of elements of $H$ for $n_{t}>0$, and $H^{0}$ is a singleton set. If $\lambda_{t}$ is an $n_{t}$-ary operation on $H$, then $n_{t}$ is called the arity (or rank) of $\lambda_{t}$.

A language (or type) of an algebra is a set $L$ such that a non-negative integer $n_{t}$ and a function symbol $\lambda_{t}$ is assigned to each member $t$ of $L$. If $L$ is a language of algebras, then a universal algebra $\mathcal{H}$ of type $L$ is an ordered pair $(H, \lambda)$, where $H$ is a non-empty set and $\lambda=\left(\lambda_{t}{ }^{H}: t \in L\right)$ is a family of operations on $H$, where $\lambda_{t}$ is an $n_{t}$-ary operation on $H$.

If there is no confusion, we denote $\lambda_{t}{ }^{H}$ by $\lambda_{t}$ and use the same notation $\lambda$ for every algebra $H$.

Definition 2.2. Let $\mathcal{H}$ and $\mathcal{H}^{\prime}$ be algebras of the same type $L$. A function $f: H \rightarrow H^{\prime}$ is called a homomorphism if for every $t \in L$ and all $h_{1}, \ldots, h_{n_{t}} \in H$,

$$
f\left(\lambda_{t}\left(h_{1}, \ldots, h_{n_{t}}\right)\right)=\lambda_{t}\left(f\left(h_{1}\right), \ldots, f\left(h_{n_{t}}\right)\right) .
$$

Definition 2.3. Let $\theta$ be an equivalence relation on a set $H$. Then $\theta$ is said to be a congruence of an algebra $\mathcal{H}$ if for each $t \in L$,

$$
\lambda_{t}\left(h_{1}, \ldots, h_{n_{t}}\right) \theta \lambda_{t}\left(h_{1}^{\prime}, \ldots, h_{n_{t}}^{\prime}\right),
$$

whenever $h_{1}, \ldots, h_{n_{t}}, h_{1}^{\prime}, \ldots, h_{n_{t}}^{\prime} \in H$ satisfy $h_{i} \theta h_{i}^{\prime}$ for $i=1, \ldots, n_{t}$.

Now, we define the "hyper" counter parts of the above notions.

Let $H$ be a non-empty set and $f$ be a mapping $f: H \times H \rightarrow \mathcal{P}^{*}(H)$, where $\mathcal{P}^{*}(H)$ is the set of all non-empty subsets of $H$. Then $f$ is called a binary hyperoperation on $H$. In general, a mapping $f: H^{n} \rightarrow \mathcal{P}^{*}(H)$ is called an $n$-ary hyperoperation and $n$ is called the order of hyperoperation.

Let $f$ be an $n$-ary hyperoperation on $H$ and $A_{1}, \ldots, A_{n}$ subsets of $H$. We define $f\left(A_{1}, \ldots, A_{n}\right)=\bigcup\left\{f\left(x_{1}, \ldots, x_{n}\right) \mid x_{i} \in A_{i}, i=1, \ldots, n\right\}$. We shall use the following abbreviated notation: the sequence $x_{i}, x_{i+1}, \ldots, x_{j}$ will be denoted by $x_{i}^{j}$. For $j<i, x_{i}^{j}$ is the empty set. In this convention $f\left(x_{1}, \ldots, x_{i}, y_{i+1}, \ldots\right.$, $\left.y_{j}, z_{j+1}, \ldots, z_{n}\right)$ will be written as $f\left(x_{1}^{i}, y_{i+1}^{j}, z_{j+1}^{n}\right)$. 
Definition 2.4. A non-empty set $H$ with an $n$-ary hyperoperation $f: H^{n} \rightarrow$ $\mathcal{P}^{*}(H)$ will be called an $n$-ary hypergroupoid and will be denoted by $(H, f)$. An $n$-ary hypergroupoid $(H, f)$ will be called an $n$-ary semihypergroup if and only if the following associative axiom holds:

$$
f\left(x_{1}^{i-1}, f\left(x_{i}^{n+i-1}\right), x_{n+i}^{2 n-1}\right)=f\left(x_{1}^{j-1}, f\left(x_{j}^{n+j-1}\right), x_{n+j}^{2 n-1}\right)
$$

for every $i, j \in\{1,2, \ldots, n\}$ and $x_{1}, x_{2}, \ldots, x_{2 n-1} \in H$.

If for all $\left(a_{1}, a_{2}, \ldots, a_{n}\right) \in H^{n}$, the set $f\left(a_{1}, a_{2}, \ldots, a_{n}\right)$ is a singleton, then $f$ is called an $n$-ary operation and $(H, f)$ is called an $n$-ary groupoid (resp. $n$-ary semigroup).

An $n$-ary semihypergroup $(H, f)$ in which the equation

$$
b \in f\left(a_{1}^{i-1}, x_{i}, a_{i+1}^{n}\right)
$$

has a solution $x_{i} \in H$ for every $a_{1}, \ldots, a_{i-1}, a_{i+1}, \ldots, a_{n}, b \in H$ and $1 \leq i \leq n$, is called an $n$-ary hypergroup. In the above definition, if $f$ is an $n$-ary operation, then the equation $(*)$ is as follows:

$$
b=f\left(a_{1}^{i-1}, x_{i}, a_{i+1}^{n}\right) .
$$

In this case $(H, f)$ is an $n$-ary group.

Let $n_{t}$ be a nonnegative integer. Then an $n_{t}$-ary hyperoperation on a nonempty set $H$ is a function $\lambda_{t}: H^{n_{t}} \rightarrow \mathcal{P}^{*}(H)$, where $\mathcal{P}^{*}(H)$ is the set of all non-empty subsets of $H$.

A universal hyperalgebra (or simply, a hyperalgebra) $\mathcal{H}$ of type $L$ is a pair $\left(H,\left(\lambda_{t}\right)_{t \in L}\right)$, where $\lambda_{t}$ is a hyperoperation on $H$ for each $t \in L$.

By a quasi-ordered semigroup we mean a triple $(G, \circ, \leq)$, where $(G, \circ)$ is a semigroup and binary relation $\leq$ is a quasi-ordering (i.e., it is reflexive and transitive) on the set $G$ such that for any triple $x, y, z \in G$ with the property $x \leq y$ also $x \circ z \leq y \circ z$ and $z \circ x \leq z \circ y$ hold. By an ordered (semi)group we mean (as usual) a triple $(G, \circ, \leq)$, where $(G, \circ)$ is a (semi)group and $\leq$ is a reflexive, antisymmetrical and transitive binary relation on $G$ such that for any triple $x, y, z \in G$ with the property $x \leq y$ also $x \circ z \leq y \circ z$ and $z \circ x \leq z \circ y$ hold.

The following proposition is a generalization of Lemma 1 in [9].

Proposition 2.5. Let $(H, \circ, \leq)$ be a quasi-ordered semigroup. Define an n-ary hyperoperation $f: H^{n} \rightarrow \mathcal{P}^{*}(H)$ by $f\left(h_{1}, \ldots, h_{n}\right)=\left\{h \in H:\left(h_{1} \circ \cdots \circ h_{n}\right) \leq h\right\}$ for all elements $h_{1}, \ldots, h_{n} \in H$. Then

(1) $(H, f)$ is an n-ary semihypergroup which is commutative if the semigroup $(H, \circ)$ is commutative.

(2) If $(H, f)$ is the above defined $n$-ary semihypergroup, then $(H, f)$ is an $n$-ary hypergroup if and only if for any elements $h_{1}, \ldots, h_{n}, b \in H$ there exist elements $c_{1}, \ldots, c_{n} \in H$ with a property $h_{1} \circ \cdots \circ h_{i-1} \circ c_{i} \circ h_{i+1} \circ \cdots \circ h_{n} \leq b$ for every $1 \leq i \leq n$. 
Proof. (1) In order to verify the associativity law, suppose that $a_{1}^{2 n-1} \in H$. Then

$$
\begin{aligned}
& f\left(a_{1}^{i-1}, f\left(a_{i}^{n+i-1}\right), a_{n+i}^{2 n-1}\right) \\
= & \bigcup_{x \in f\left(a_{i}^{n+i-1}\right)} f\left(a_{1}^{i-1}, x, a_{n+i}^{2 n-1}\right) \\
= & \bigcup_{a_{i} \circ \cdots \circ a_{n+i-1} \leq x}\left\{y: a_{1} \circ \cdots \circ a_{i-1} \circ x \circ a_{n+i} \circ \cdots \circ a_{2 n-1} \leq y\right\} \\
= & \left\{y: a_{1} \circ \cdots \circ a_{2 n-1} \leq y\right\} \\
= & \left\{y: a_{1} \circ \ldots a_{j-1} \circ\left(a_{j} \circ \cdots \circ a_{n+j-1}\right) \circ \cdots \circ a_{2 n-1} \leq y\right\} \\
= & \bigcup_{a_{j} \circ \cdots \circ a_{n+j-1} \leq z}\left\{y: a_{1} \circ \cdots \circ a_{j-1} \circ z \circ a_{n+j} \circ \cdots \circ a_{2 n-1} \leq y\right\} \\
= & \bigcup_{x \in f\left(a_{j}^{n+j-1}\right)} f\left(a_{1}^{j-1}, x, a_{n+j}^{2 n-1}\right) f\left(a_{1}^{j-1}, f\left(a_{j}^{n+j-1}\right), a_{n+j}^{2 n-1}\right) .
\end{aligned}
$$

Therefore $(H, f)$ is an $n$-ary semihypergroup. Evidently, if the $\operatorname{semigroup~}(H, \circ)$ is commutative, then the $n$-ary semihypergroup $(H, f)$ is also commutative.

(2) It is clear.

Corollary 2.6. Let $(H, \circ, \leq)$ be an ordered group. Define an $n$-ary hyperoperation $f: H^{n} \rightarrow \mathcal{P}^{*}(H)$ by $f\left(h_{1}, \ldots, h_{n}\right)=\left\{h \in H:\left(h_{1} \circ \cdots \circ h_{n}\right) \leq h\right\}$ for all elements $h_{1}, \ldots, h_{n} \in H$. Then $(H, f)$ is an $n$-ary hypergroup which is commutative if the group $(H, \cdot)$ is commutative.

Proof. By Proposition 2.5(1), $(H, f)$ is an $n$-ary semihypergroup. Suppose that $a_{1}, \ldots, a_{n}, b$ are arbitrary elements of $H$. We denote

$$
x_{i}=a_{i-1}^{-1} \circ \cdots \circ a_{1}^{-1} \circ b \circ a_{n}^{-1} \circ \cdots \circ a_{n+i}^{-1} .
$$

Then $b=a_{1} \circ \cdots \circ a_{i-1} \circ x_{i} \circ a_{n+i} \circ \cdots \circ a_{n}$. Thus $a_{1} \circ \cdots \circ a_{i-1} \circ x_{i} \circ a_{n+i} \circ \cdots \circ a_{n} \leq b$, and so the equation $b \in f\left(a_{1}^{i-1}, x_{i}, a_{n+i}^{n}\right)$ has a solution $x_{i}$.

\section{Some examples of hyperalgebra}

\subsection{Constructions of $\boldsymbol{n}$-ary hyperoperators on ordered groups}

In this paragraph, we give a generalization for [9]. A Fredholm-Voltra integral operator can be written as the following:

$$
\begin{aligned}
F(\lambda, \mu, K, L, f): & C(J \times[0,+\infty)) \longrightarrow C(J \times[0,+\infty)) \\
& \varphi(x, t) \mapsto F(\lambda, \mu, K(x, t, s), L(x, t, \tau), f(x, t))(\varphi(x, t)) \\
= & \lambda \int_{a}^{b} K(x, t, s) \varphi(s, t) d s+\mu \int_{0}^{t} L(x, t, \tau) \varphi(x, \tau) d \tau+f(x, t),
\end{aligned}
$$

where $J=(a, b), K(x, t, s) \in C(J \times[0,+\infty) \times J), L(x, t, \tau) \in C(J \times[0,+\infty)$, $[0, t])$, (kernels), are a real or complex valued functions (mostly positive real 
functions), $f(x, t) \in C(J, \times[0,+\infty)), \lambda, \mu$ are two real numerical parameters. Usually there are considered Fredholm-Voltra integral equations with a nondegenerate Lebesgue square integrable kernels $K(x, t, s)$ and $L(x, t, \tau)$. In this contribution we will construct an $n$-ary hypergroup on the set of operators $F(\lambda, \mu, K(x, t, s), L(x, t, \tau), f(x, t))$ with continuous functions $K, L, f$ and two nonzero parameters $\lambda, \mu$. For our purposes we will consider continuous positive functions only, in order to avoid some obstacles with integrability of functions in the form of fractions.

Denote $F V=\left\{F(\lambda, \mu, K(x, t, s), L(x, t, \tau), f(x, t)): \lambda, \mu \in \mathbb{R}, \lambda^{2}+\mu^{2} \neq\right.$ $0, K(x, t, s) \in C(J \times[0,+\infty) \times J), L(x, t, \tau) \in C(J \times[0,+\infty),[0, t]), f(x, t) \in$ $C(J, \times[0,+\infty))\}$.

For any pairs of operators $F\left(\lambda_{1}, \mu_{1}, K_{1}, L_{1}, f_{1}\right), F\left(\lambda_{2}, \mu_{2}, K_{2}, L_{2}, f_{2}\right)$ in $F V$ let us define

$$
\begin{aligned}
& F\left(\lambda_{1}, \mu_{1}, K_{1}, L_{1}, f_{1}\right) \circ F\left(\lambda_{2}, \mu_{2}, K_{2}, L_{2}, f_{2}\right) \\
= & F\left(\lambda_{1} \lambda_{2}, \mu_{1} \mu_{2}, K_{2} f_{1}+K_{1}, L_{2} f_{1}+L_{1}, f_{1} f_{2}\right)
\end{aligned}
$$

and $F\left(\lambda_{1}, \mu_{1}, K_{1}, L_{1}, f_{1}\right) \leq F\left(\lambda_{2}, \mu_{2}, K_{2}, L_{2}, f_{2}\right)$ if and only if $\lambda_{1}=\lambda_{2}, \mu_{1}=$ $\mu_{2}, f_{1}(x, t) \equiv f_{2}(x, t)$ for any $(x, t) \in J \times[0, \infty), K_{1}(x, t, s) \leq K_{2}(x, t, s)$ for any $(x, t, s) \in(J \times[0,+\infty) \times J), L_{1}(x, t, \tau) \leq L_{2}(x, t, \tau)$ for any $(x, t, \tau) \in$ $(J \times[0,+\infty),[0, t])$.

Proposition 3.1.1. $(F V, \circ, \leq)$ is a noncommutative ordered group.

Proof. Suppose that $F\left(\lambda_{i}, \mu_{i}, K_{i}, L_{i}, f_{i}\right) \in F V, i=1,2,3$. Then

$$
\begin{aligned}
& F\left(\lambda_{1}, \mu_{1}, K_{1}, L_{1}, f_{1}\right) \circ\left(F\left(\lambda_{2}, \mu_{2}, K_{2}, L_{2}, f_{2}\right) \circ F\left(\lambda_{3}, \mu_{3}, K_{3}, L_{3}, f_{3}\right)\right) \\
= & F\left(\lambda_{1}, \mu_{1}, K_{1}, L_{1}, f_{1}\right) \circ\left(F\left(\lambda_{2} \lambda_{3}, \mu_{2} \mu_{3}, K_{3} f_{2}+K_{2}, L_{3} f_{2}+L_{2}, f_{2} f_{3}\right)\right. \\
= & F\left(\lambda_{1} \lambda_{2} \lambda_{3}, \mu_{1} \mu_{2} \mu_{3}, K_{3} f_{2} f_{1}+K_{2} f_{1}+K_{1}, L_{3} f_{2} f_{1}+L_{2} f_{1}+L_{1}, f_{1} f_{2} f_{3}\right) \\
= & F\left(\lambda_{1} \lambda_{2}, \mu_{1} \mu_{2}, K_{2} f_{1}+K_{1}, L_{2} f_{1}+L_{1}, f_{1} f_{2}\right) \circ\left(F\left(\lambda_{3}, \mu_{3}, K_{3}, L_{3}, f_{3}\right)\right) \\
= & \left(F\left(\lambda_{1}, \mu_{1}, K_{1}, L_{1}, f_{1}\right) \circ F\left(\lambda_{2}, \mu_{2}, K_{2}, L_{2}, f_{2}\right)\right) \circ F\left(\lambda_{3}, \mu_{3}, K_{3}, L_{3}, f_{3}\right) .
\end{aligned}
$$

Thus the binary operation " $\circ$ " is associative. Further, any operator $F(\lambda, \mu$, $K, L, f) \in F V$ and operator $F(1,1,0,0,1)$ satisfy an equality

$$
\begin{aligned}
F(\lambda, \mu, K, L, f) \circ F(1,1,0,0,1) & =F(\lambda, \mu, K, L, f) \\
& =F(1,1,0,0,1) \circ F(\lambda, \mu, K, L, f) .
\end{aligned}
$$

Thus the operator $F(1,1,0,0,1)$ is unit of the semigroup $(F V, \circ)$.

Now, since for any operator $F(\lambda, \mu, K, L, f) \in F V$ there holds $\lambda^{2}+\mu^{2} \neq$ 0 and $f(x, t)>0$ for all $(x, t) \in J \times[0,+\infty)$. We have that the operator $F\left(\frac{1}{\lambda}, \frac{1}{\mu}, \frac{-K}{f}, \frac{-L}{f}, \frac{1}{f}\right)$ is well defined and belong to $F V$. Then,

$$
\begin{aligned}
& F\left(\frac{1}{\lambda}, \frac{1}{\mu}, \frac{-K}{f}, \frac{-L}{f}, \frac{1}{f}\right) \circ F(\lambda, \mu, K, L, f)=F(1,1,0,0,1), \\
& F(\lambda, \mu, K, L, f) \circ F\left(\frac{1}{\lambda}, \frac{1}{\mu}, \frac{-K}{f}, \frac{-L}{f}, \frac{1}{f}\right)=F(1,1,0,0,1),
\end{aligned}
$$


which means that $F^{-1}(\lambda, \mu, K, L, f)=F\left(\frac{1}{\lambda}, \frac{1}{\mu}, \frac{-K}{f}, \frac{-L}{f}, \frac{1}{f}\right)$, hence $(F V, \circ)$ is a group. Evidently the binary operation "o" is non-commutative on " $F V$ ". From the definition of the relation " $\leq$ " it follows immediately that this relation is reflexive, anti symmetrical and transitive on $F V$. Hence the pair $(F V, \leq)$ is an ordered set. It remain that to verify the compatibility of the ordering " $\leq$ " on $F V$ with the binary operation "о". Suppose that $F\left(\lambda_{1}, \mu_{1}, K_{1}, L_{1}, f_{1}\right), F\left(\lambda_{2}\right.$, $\left.\mu_{2}, K_{2}, L_{2}, f_{2}\right) \in F V$ are integral operators satisfying

$$
F\left(\lambda_{1}, \mu_{1}, K_{1}, L_{1}, f_{1}\right) \leq F\left(\lambda_{2}, \mu_{2}, K_{2}, L_{2}, f_{2}\right),
$$

and $F(\lambda, \mu, K, L, f) \in F V$ is an arbitrary operator. Then

$$
\begin{array}{r}
0<f_{1}(x, t) \equiv f_{2}(x, t), \\
0 \neq \lambda_{1}=\lambda_{2}, 0 \neq \mu_{1}=\mu_{2}, \\
K_{1}(x, t, s) \leq K_{2}(x, t, s), \\
L_{1}(x, t, \tau) \leq L_{2}(x, t, \tau)
\end{array}
$$

for any $x \in J, t \in[0, \infty), s \in J, \tau \in[0, t]$. Hence

$$
\begin{array}{r}
\lambda \lambda_{1}=\lambda \lambda_{2}, \quad f(x, t) f_{1}(x, t) \equiv f(x, t) f_{2}(x, t), \\
K_{1}(x, t, s) f(x, t)+K(x, t, s) \leq K_{2}(x, t, s) f(x, t)+K(x, t, s), \\
L(x, t, s) f_{1}(x, t)+K_{1}(x, t, s) \leq K(x, t, s) f_{2}(x, t)+K_{2}(x, t, s), \\
L_{1}(x, t, \tau) f(x, t)+L(x, t, \tau) \leq L_{2}(x, t, \tau) f(x, t)+K(x, t, \tau), \\
L(x, t, \tau) f_{1}(x, t)+K_{1}(x, t, \tau) \leq L(x, t, \tau) f_{2}(x, t)+L_{2}(x, t, \tau),
\end{array}
$$

and so

$$
\begin{aligned}
F(\lambda, \mu, K, L, f) \circ F\left(\lambda_{1}, \mu_{1}, K_{1}, L_{1}, f_{1}\right) & =F\left(\lambda \lambda_{1}, \mu_{1} \mu_{2}, K_{1} f+K, L_{1} f+L, f f_{1}\right) \\
& \leq F\left(\lambda \lambda_{2}, \mu \mu_{2}, K_{2} f+K, L_{2} f+L, f f_{2}\right) \\
& =F(\lambda, \mu, K, L, f) \circ F\left(\lambda_{2}, \mu_{2}, K_{2}, L_{2}, f_{2}\right)
\end{aligned}
$$

and

$$
\begin{aligned}
& F\left(\lambda_{1}, \mu_{1}, K_{1}, L_{1}, f_{1}\right) \circ F(\lambda, \mu, K, L, f) \\
= & F\left(\lambda_{1} \lambda, \mu_{1} \mu, K f_{1}+K_{1}, L f_{1}+L_{1}, f_{1} f\right) \\
\leq & F\left(\lambda_{2} \lambda_{1}, \mu_{2} \mu, K f_{2}+K_{2}, L f_{2}+L_{2}, f_{2} f\right) \\
= & F\left(\lambda_{2}, \mu_{2}, K_{2}, L_{2}, f_{2}\right) \circ F(\lambda, \mu, K, L, f) .
\end{aligned}
$$

Consequently, $(F V, \circ, \leq)$ is a noncommutative ordered group.

\subsection{About the definition of copula}

The mathematical concept of copulas has been known in principle for about half a century. As early as 1959, Sklar published his work now referred to in the literature as Sklars theorem which is of central significance when one is applying copulas to statistical investigations of the relation between marginal distributions and their dependence structure. However, it took about 
four decades before copulas became more widely used by the mathematical community. Since the late 1990s, copulas have been rediscovered, becoming more and more attractive in statistics. Consequently, the amount of literature in this area has also increased. A good example of this is Nelsens excellent text An Introduction to Copulas, which was first published in 1999 and has since been reissued in a revised format. Today, the book is firmly established as the standard reference in the area of copulas (see [16]).

During a long time statisticians have been interested on the relationship between a multivariate distribution function and its lower dimensional margins. M. Frchet, and G. Dall'Aglio did some interesting works about this matter in the fifties, studying the bivariate and trivariate distribution functions with given univariate margins. The answer to this problem for the univariate margins case was given by A. Sklar in 1959 creating a new class of functions which he called copulas. These new functions are restrictions to $[0,1]^{2}$ of bivariate distribution functions whose margins are uniform in $[0,1]$. In short, Sklar showed that if $H$ is a bivariate distribution function whit margins $F(x)$ and $G(y)$, then there exists a copula $C$ such that $H(x, y)=C(F(x), G(y))$. The recent book by R. B. Nelsen (see [16]) is an important monograph about copulas.

Definition 3.2.1 (Copula). A $d$-dimensional copula is a function $C:[0,1]^{d} \rightarrow$ $[0,1]$ and having the following properties:

(i) let $x \in[0,1]^{d}$, thus $x=\left(x_{1}, \ldots, x_{d}\right)$, where $x_{j} \in[0,1], \forall j \in\{1, \ldots, d\}$, then $C(x)$ is increasing in each component,

(ii) $C(x)=0$ if at least one coordinate $x_{j}=0$,

(iii) $C(x)=x_{k}$ if $x_{j}=1, \forall j \neq k$,

(iv) for every $i=1, \ldots, d$, and $a_{i}, b_{i} \in[0,1]$ with $a_{i} \leq b_{i}$ and a hypercube $B=[a, b]=\left[a_{1}, b_{1}\right] \times\left[a_{2}, b_{2}\right] \times \cdots \times\left[a_{d}, b_{d}\right]$ whose vertices lie in the domain of $C$, we have volume $V_{C}(B) \geq 0$, where $V_{C}(B)$ is defined as follows,

$$
V_{C}(B)=\sum_{i_{1}=1}^{2} \ldots \sum_{i_{d}=1}^{2}(-1)^{i_{1}+\cdots+i_{d}} C\left(x_{1, i_{1}}, \ldots, x_{d, i_{d}}\right)
$$

for all $x_{1,1}, \ldots, x_{d, 1} \in[0,1]$ and $x_{1,2}, \ldots, x_{d, 2} \in[0,1]$ with $x_{j, 1} \leq$ $x_{j, 2},(1 \leq j \leq d)$.

Theorem 3.2.2 (Sklar's theorem, [18]). Let $F$ be a d-dimensional distribution function with margins $F_{1}, F_{2}, \ldots, F_{d}$. Then there exits an d-copula $C$ such that for all $x_{1}, \ldots, x_{d} \in \mathbb{R}^{d}, F\left(x_{1}, \ldots, x_{d}\right)=C\left(F_{1}\left(x_{1}\right), \ldots, F_{d}\left(x_{d}\right)\right)$. If $F_{1}, \ldots, F_{d}$ are continuous, then $C$ is unique; otherwise, $C$ is uniquely determined on $\operatorname{Ran}\left(F_{1}\right) \times \cdots \times \operatorname{Ran}\left(F_{d}\right)$. Conversely, if $C$ is an d-copula and dimensional distribution function with margins $F_{1}, \ldots, F_{d}$. Here, $\operatorname{Ran}(F)$ indicates range of the function $F$. 
Let $\mathcal{C}_{d}$ denote the set of all $d$-dimensional copulas. Denote by $\partial_{i} C$ the partial derivative of $C \in \mathcal{C}_{d}$ with respect to the $i$-th variable.

\section{Example 3.2.3.}

(1) The $\mathcal{C}_{2}$ can be equipped with the $*$-multiplication (see [16]), defined by;

$$
\begin{aligned}
& A * B:[0,1]^{2} \rightarrow[0,1] \text { for all } A, B \in \mathbb{C}_{2} \\
& (A * B)(x, y)=\int_{0}^{1} \partial_{2} A(x, t) \partial_{1} B(t, y) d t
\end{aligned}
$$

Notice that $(A * B)(x, y)$ is well defined because each partial derivative is an $L^{1}$-function with respect to $t$, as well as $L^{\infty}$-function. The set of copulas is a non-commutative semigroup under the operation $*$. For all $\left(x_{1}, \ldots, x_{n}\right) \in I^{n}$, where $C^{+}$is the so-called Fréchet-Hoeffding Upper given by $C^{+}\left(x_{1}, \ldots, x_{n}\right)=\min \left\{x_{1}, \ldots, x_{n}\right\}$. Direct calculations show that for any copula $C \in \mathcal{C}_{2}$ we have $C^{+} * C=C * C^{+}=C$. The copula $C^{+}$represents the unit element in $\left(\mathcal{C}_{2}, *\right)$.

(2) Here, we define a generalization of the $*$ product over the copulas. Let $A \in \mathcal{C}_{m}$ and $B \in \mathcal{C}_{n}$. We can define the $(m+n-1)$-copula by the following;

$$
\begin{gathered}
A \star B:[0,1]^{m+n-1} \longrightarrow[0,1] \\
(A \star B)\left(x_{1}, \ldots, x_{m+n-1}\right) \\
=\int_{0}^{x_{m}} \partial_{m} A\left(x_{1}, \ldots, x_{m-1}, t\right) \partial_{1} B\left(t, x_{m+1}, \ldots, x_{m+n-1}\right) d t .
\end{gathered}
$$

Observe, if $m=n=2$, the $\star$ and $*$ products are related by

$$
(A * B)(x, y)=(A \star B)(x, 1, y) .
$$

By arguments similar to those used in [16], it is readily verified that $A \star B$ is $(m+n-1)$-copula and that the $*$ product distributive over convex combinations, is associative and continuous in each place.

Now, we introduce a new construction of copulas.

Proposition 3.2.4. Let $A$ and $B$ be two d-copulas in $\mathcal{C}_{2}$ and let $\mathcal{C}=\left\{C_{l}\right\}_{l \in L}$ be a family in $\mathcal{C}_{d}$. The the mapping $\left(A \star_{\mathcal{C}} B\right):[0,1]^{d} \rightarrow \mathcal{P}^{*}([0,1])$ defined by

$$
\begin{aligned}
& \left(A *_{\mathcal{C}} B\right)\left(x_{1}, \ldots, x_{d}\right) \\
= & \bigcup_{l \in L} \bigcup_{i+j=d+1} \int_{0}^{1} C_{l}\left(\partial_{i} A\left(x_{1}, \ldots, x_{i-1}, t, x_{i+1} \ldots, x_{d}\right), \partial_{j} B\left(x_{1}, \ldots, x_{j-1}, t, x_{j+1} \ldots, x_{d}\right)\right) d t .
\end{aligned}
$$

is an $n$-ary hyperoperation.

Proof. The proposition can be obtained from definitions and the proof is omitted. 


\section{Topological hyperdynamical systems}

Let $(H, \tau)$ be a topological space and $\left(H,\left(\lambda_{t}\right)_{t \in L}\right)$ be a hyperalgebra, where $\lambda_{t}: H^{n_{t}} \rightarrow \mathcal{P}^{*}(H)$.

Definition 4.1. The hyperoperation $\lambda_{t}$ is called;

(1) pseudo-continuous or for short $p$-continuous, if every $O \in \tau$ the set $O_{\lambda_{t}}=\left\{\left(x_{1}, \ldots, x_{n}\right) \in H^{n}: \lambda_{t}\left(x_{1}, \ldots, x_{n}\right) \subseteq O\right\}$ is open in $H^{n}$,

(2) strongly pseudo-continuous or for short sp-continuous, if for every $O \in$ $\tau$ the set $O_{\lambda_{t}}=\left\{\left(x_{1}, \ldots, x_{n}\right) \in H^{n}: \lambda_{t}\left(x_{1}, \ldots, x_{n}\right)=O\right\}$ is open in $H^{n}$.

We denote by $\tau^{n}$ the Cartesian product $\tau \times \cdots \times \tau$, where $\tau$ appears $n$ times.

Definition 4.2. Let $\left(H,\left(\lambda_{t}\right)_{t \in L}\right)$ be a hyperalgebra, where $\lambda_{t}: H^{n_{t}} \rightarrow \mathcal{P}^{*}(H)$. Let $(H, \tau)$ be a topological space and $\tau_{*}$ be a topology on $\mathcal{P}^{*}(H)$.

(1) The hyperoperation $\lambda_{t}$ is called $\tau_{*}$-continuous if the mapping $\lambda_{t}$ is continuous with respect to topologies $\tau^{n}$ and $\tau_{*}$.

(2) The triple $\left(H,\left(\lambda_{t}\right)_{t \in L}, \tau\right)$ is called a pseudo-topological (strongly pseudo-topological) hypersystem, if the hyperoperations $\lambda_{t}$ for all $t \in L$, are pseudo-continuous (strongly pseudo-continuous).

(3) The quadruple $\left(H,\left(\lambda_{t}\right)_{t \in L}, \tau, \tau_{*}\right)$ is called $\tau_{*}$-topological hypersystem, if the hyperoperation $\left(\lambda_{t}\right)_{t \in L}$ is $\tau_{*}$-continuous.

Now, we will show that for a given topology $\tau$ on $H$, it is possible to find a topology $\tau_{*}$ in such a way that $\tau_{*}$-continuity means just $p$-continuity or $s p$ continuity, respectively.

Proposition 4.3. Let $(H, \tau)$ be a topological space. Then the family $\mathcal{U}$ consisting of all sets $S_{v}=\left\{u \in \mathcal{P}^{*}(H): u \subseteq v\right\}, v \in \tau$, is a base of a topology $\tau_{\mathcal{U}}$ on $\mathcal{P}^{*}(H)$.

Proof. Let $S_{v_{1}}, S_{v_{2}} \in \mathcal{U}, v_{1}, v_{2} \in \tau$. Evidently, $S_{v_{1}} \cap S_{v_{2}}=S_{v_{1} \cap v_{2}}$ as $v_{1} \cap v_{2} \in \tau$. Thus for every point $h \in S_{v_{1}} \cap S_{v_{2}}$ there exists $S_{v_{1} \cap v_{2}}$ such that $S_{v_{1} \cap v_{2}} \subseteq$ $S_{v_{1}} \cap S_{v_{2}}$. Further, for every $h \in H$ there exists a $S_{H}=\mathcal{P}^{*}(H)($ Here $H \in \tau)$ such that $h \in S_{H}$.

The topology $T_{\mathcal{U}}$ on $\mathcal{P}^{*}(H)$ in Proposition 4.3 is called an upper topology on $\mathcal{P}^{*}(H)$ induced by the topology $\tau$ on $H$.

Theorem 4.4. Let $\left(H,\left(\lambda_{n_{t}}\right)_{t \in L}\right)$ be a hyperalgebra and $(H, \tau)$ be a topological space. Then $\left(H,\left(\lambda_{n_{t}}\right)_{t \in L}\right)$ is a pseudo-topological hypersystem if and only if the quadruple $\left(H,\left(\lambda_{n_{t}}\right)_{t \in L}, \tau, \tau_{\mathcal{U}}\right)$ is $\tau_{\mathcal{U}}$-topological hypersystem.

Proof. For an open set $v \in \tau$ we have:

$$
\begin{aligned}
\lambda_{n_{t}}^{-1}\left(S_{v}\right) & =\left\{\left(h_{1}, \ldots, h_{n_{t}}\right) \in H^{n_{t}}: \lambda_{n_{t}}\left(h_{1}, \ldots, h_{n_{t}}\right) \in S_{v}\right\} \\
& =\left\{\left(h_{1}, \ldots, h_{n_{t}}\right) \in H^{n_{t}}: \lambda_{n_{t}}\left(h_{1}, \ldots, h_{n_{t}}\right) \subseteq v\right\}=V_{*} .
\end{aligned}
$$

Therefore $\lambda_{n_{t}}$ is continuous if and only if $V_{*}$ is open for any $v \in \tau$, i.e., when $\lambda_{n_{t}}$ is $p$-continuous. 
Proposition 4.5. Let $(H, \tau)$ be a topological space. Then the family $\mathcal{F}$ consisting of all sets

$$
I_{v}=\left\{u \in \mathcal{P}^{*}(H): u \approx v\right\}, \quad v \in \tau,
$$

is a subbase of topology $\tau_{\mathcal{F}}$ on $\mathcal{P}^{*}(H)$, where $u \approx v$ means $u \cap v \neq \emptyset$.

Proof. It is sufficient to verify that $\bigcup_{v \in \tau} I_{v}=\mathcal{P}^{*}(H)$ which is evident as $H \in \tau$ and $I_{H}=\mathcal{P}^{*}(H)$.

Definition 4.6. The topology $\tau_{\mathcal{F}}$ on $\mathcal{P}^{*}(H)$ in Proposition 4.5 is called a lower topology on $\mathcal{P}^{*}(H)$ induced by the topology $\tau$ on $H$.

Theorem 4.7. Let $\left(H,\left(\lambda_{n_{t}}\right)_{t \in L}\right)$ be a hyperalgebra and $(H, \tau)$ be a topological space. Then $\left(H,\left(\lambda_{n_{t}}\right)_{t \in L, \tau}\right)$ is a strongly pseudo-topological hypersystem if and only if the quadruple $\left(H,\left(\lambda_{n_{t}}\right)_{t \in L}, \tau, \tau_{\mathcal{F}}\right)$ is $\tau_{\mathcal{F}}$-topological hypersystem.

Proof. For an open set $v \in \tau$ we have:

$$
\begin{aligned}
\lambda_{n_{t}}^{-1}\left(I_{v}\right) & =\left\{\left(h_{1}, \ldots, h_{n_{t}}\right) \in H^{n_{t}}: \lambda_{n_{t}}\left(h_{1}, \ldots, h_{n_{t}}\right) \in I_{v}\right\} \\
& =\left\{\left(h_{1}, \ldots, h_{n_{t}}\right) \in H^{n_{t}}: \lambda_{n_{t}}\left(h_{1}, \ldots, h_{n_{t}}\right) \approx v\right\}=V^{*} .
\end{aligned}
$$

Therefore $\lambda_{n_{t}}$ is continuous if and only if $V^{*}$ is open for any $v \in \tau$, i.e., when $\lambda_{n_{t}}$ is $s p$-continuous.

In 1922 an Austrian mathematician, L. Vietoris [19] defined on the collection of all non-empty closed subsets $C l(H)$ of a topological space $(H, \tau)$ a topology as follows: for each finite collection $U_{1}, \ldots, U_{k} \in \tau$, let $\left\langle U_{1}, \ldots, U_{k}\right\rangle$ denote the set of all $s \in C l(H)$ such that $S \subseteq \bigcup_{i=1}^{k}$ and $S \cap U_{i} \neq \emptyset$ for each $i=1,2, \ldots, k$. The sets $\left\langle U_{1}, \ldots, U_{k}\right\rangle$ form the base of a topology on $C l(H)$. This approach can be applied to the collection of all non-empty subsets of $(H, \tau)$ as follows:

Proposition 4.8. Let $(H, \tau)$ be a topological space. For any $u_{1}, \ldots, u_{k} \in \tau, k \in$ $\mathbb{N}$, let us denote

$$
\mathcal{V}\left(u_{1}, \ldots, u_{k}\right)=\left\{B \in \mathcal{P}^{*}(H): B \subseteq \bigcup_{i=1}^{k} u_{i} \text { and } B \approx u_{i} ; \text { for } i=1, \ldots, k\right\} .
$$

The family $\mathcal{B}$ of all set $\mathcal{V}\left(u_{1}, \ldots, u_{k}\right)$ forms a base of a topological space $\left(\mathcal{P}^{*}(H)\right.$, $\tau \mathcal{V})$.

The topology $\tau_{\mathcal{V}}$ in Proposition 4.8 is called a Vietoris topology on $\mathcal{P}^{*}(H)$.

The vietories topology is one of the possible ways how to define a topology $\mathcal{P}^{*}(H)$ if a topology on $H$ is given.

Proposition 4.9. Vietoris topology $\tau_{\mathcal{V}}$ is the lowest common refinement of upper and lower topologies $\tau_{\mathcal{U}}$ and $\tau_{\mathcal{F}}$.

Theorem 4.10. Let $(H, \tau)$ be a topological space and $\left(H,\left(\lambda_{n_{t}}\right)_{t \in L}\right)$ be a hyperalgebra. The triple $\left(H,\left(\lambda_{n_{t}}\right)_{t \in L}, \tau\right)$ is both pseudotopological and strongly pseudotopological hypersystem if and only if the quadruple $\left(H,\left(\lambda_{n_{t}}\right)_{t \in L}, \tau, \tau_{\mathcal{V}}\right)$ is $\tau_{\mathcal{V}}$ - hypersystem.

Proof. The proof is evident. 


\section{Universal hyperdynamical systems}

A universal hyperdynamical system $\Phi$ of type $\tau$ is a triple $(X, \Phi, \mathcal{H})$, where $\mathcal{H}=\left(H,\left(\lambda_{n_{t}}\right)_{t \in L}\right)$ is a hyperalgebra of type $L, X$ is a non-empty set and $\Phi$ is a map $\Phi: H \times X \rightarrow \mathcal{P}^{*}(H)$ with the following property;

If $h_{1}, \ldots, h_{n_{t}} \in H$ and $x \in X$, then

$$
\left.\Phi\left(h_{1}, \Phi\left(h_{2}, \Phi\left(\ldots, \Phi\left(h_{n_{t}}, x\right), \ldots\right)\right)\right) \in \Phi\left(\lambda_{n_{t}}\right)\left(h_{1}, \ldots, h_{n_{t}}\right), x\right),
$$

where $\Phi\left(\lambda_{n_{t}}\left(h_{1}, \ldots, h_{n_{t}}\right), x\right)=\left\{\Phi(g, x): g \in \lambda_{n_{t}}\left(h_{1}, \ldots, h_{n_{t}}\right)\right\}$.

Definition 5.1. Let $\left(H,\left(\lambda_{n_{t}}\right)_{t \in L}\right)$ and $\left(H^{\prime},\left(\lambda_{n_{t}}\right)_{t \in L}^{\prime}\right)$ be two hyperalgebras of type $L$. Two universal hyperdynamical systems $(X, \Phi, \mathcal{H})$ and $\left(X^{\prime}, \Phi^{\prime}, \mathcal{H}^{\prime}\right)$ of type $L$, are called conjugate universal hyperdynamical systems if there exist one to one and onto maps $T: X \rightarrow X^{\prime}$ and $\varphi: H \rightarrow H^{\prime}$ such that the following tow axioms hold;

(1) $\varphi\left(\lambda_{n_{t}}\left(h_{1}, \ldots, h_{n_{t}}\right)\right)=\lambda^{\prime}{ }_{n_{t}}\left(\varphi\left(h_{1}\right), \ldots, \varphi\left(h_{n_{t}}\right)\right)$ for all $h_{1}, \ldots, h_{n_{t}} \in H$,

(2) $T(\Phi(h, x))=\Phi^{\prime}(\varphi(h), T(x))$ for all $h \in H$ and $x \in X$.

Theorem 5.2. Let $(T, \varphi)$ be a conjugate relation between $(X, \Phi, \mathcal{H})$ and $\left(X^{\prime}\right.$, $\left.\Phi^{\prime}, \mathcal{H}^{\prime}\right)$ and $\left(T^{\prime}, \varphi^{\prime}\right)$ be conjugate relation between $\left(X^{\prime}, \Phi^{\prime}, \mathcal{H}^{\prime}\right)$ and $\left(X^{\prime \prime}, \Phi^{\prime \prime}\right.$, $\left.\mathcal{H}^{\prime \prime}\right)$, where all universal hyperdynamical systems are of type $L$. Then

(1) the relation $\left(T^{-1}, \varphi^{-1}\right)$ is a conjugate relation between $\left(X^{\prime}, \Phi^{\prime}, \mathcal{H}^{\prime}\right)$ and $(X, \Phi, \mathcal{H})$,

(2) the relation $\left(T \circ T^{\prime}, \varphi^{\prime} \circ \varphi\right)$ is a conjugate relation between $(X, \Phi, \mathcal{H})$ and $\left(X^{\prime \prime}, \Phi^{\prime \prime}, \mathcal{H}^{\prime \prime}\right)$.

Proof. (1) If $h_{1}^{\prime}, \ldots, h_{n_{t}}^{\prime} \in H^{\prime}$, then

$$
\begin{aligned}
\varphi^{-1}\left(\lambda_{n_{t}}^{\prime}\left(h_{1}^{\prime}, \ldots, h_{n_{t}}^{\prime}\right)\right) & =\varphi^{-1}\left(\lambda^{\prime}{ }_{n_{t}}\left(\varphi\left(h_{1}\right), \ldots, \varphi\left(h_{n_{t}}\right)\right)\right) \\
& =\varphi^{-1}\left(\varphi \lambda_{n_{t}}\left(h_{1}, \ldots, h_{n_{t}}\right)\right) \\
& =\lambda_{n_{t}}\left(h_{1}, \ldots, h_{n_{t}}\right) \\
& =\lambda_{n_{t}}\left(\varphi^{-1}\left(\varphi\left(h_{1}\right)\right), \ldots, \varphi^{-1}\left(\varphi\left(h_{1}\right)_{n_{t}}\right)\right) \\
& =\lambda_{n_{t}}\left(\varphi^{-1}\left(h_{1}^{\prime}\right), \ldots, \varphi^{-1}\left(h_{n_{t}}\right)^{\prime}\right),
\end{aligned}
$$

where $h_{1}, \ldots, h_{n_{t}} \in H$. For all $h^{\prime} \in H^{\prime}$ and $x^{\prime} \in X^{\prime}$, we have

$$
\begin{aligned}
T\left(\Phi\left(\varphi^{-1}\left(h^{\prime}\right), T^{-1}\left(x^{\prime}\right)\right)\right. & =\Phi^{\prime}\left(\varphi\left(\varphi^{-1}\left(h^{\prime}\right), T\left(T^{-1}\left(x^{\prime}\right)\right)\right)\right) \\
& =\Phi^{\prime}\left(h^{\prime}, x^{\prime}\right),
\end{aligned}
$$

so $\Phi\left(\varphi^{-1}\left(h^{\prime}\right), T^{-1}\left(x^{\prime}\right)\right)=T^{-1}\left(\Phi^{\prime}\left(h^{\prime}, x^{\prime}\right)\right)$.

(2) For all $h \in H$ and $x \in X$, we have

$$
\begin{aligned}
\Phi^{\prime \prime}\left(\varphi^{\prime}(\varphi(h)), T^{\prime}\left(T\left(x^{\prime}\right)\right)\right) & =T^{\prime}\left(\Phi^{\prime}(\varphi(h), T(x))\right) \\
& =T^{\prime}\left(T\left(\Phi^{\prime}(h, x)\right)\right) \\
& =\left(T^{\prime} \circ T\right)\left(\Phi^{\prime}(h, x)\right) .
\end{aligned}
$$



$H\}$.

For any point $x \in X$, the hyperorbit of $x$ is the set $O^{H}(x)=\{\Phi(h, x): h \in$

Proposition 5.3. The set $O^{H}(x)$ is a universal hyperalgebra of type L, where $\lambda_{n_{t}}$ is a hyperoperation on $H$ for each $t \in L$.

Theorem 5.4. If $(X, \Phi, \mathcal{H})$ and $\left(X^{\prime}, \Phi^{\prime}, \mathcal{H}^{\prime}\right)$ are conjugate under $(T, \varphi)$, then $T\left(O^{H}(x)\right)=O^{H^{\prime}}(T(x))$.

Proof. If $y \in T\left(O^{H}(x)\right)$, then there exists $h \in H$ such that

$$
y \in T(\Phi(h, x))=\Phi^{\prime}(\varphi(h), T(x)) \in O^{H^{\prime}}(T(x)) .
$$

Since conjugate relation is an equivalence relation, so the first part of the proof shows that $T^{-1}\left(O^{H^{\prime}}(T(x))\right) \subseteq O^{H}(x)$. Thus $O^{H^{\prime}}(T(x)) \subseteq T\left(O^{H}(x)\right)$.

The subset $A \subseteq X$ is called invariant, if $\Phi(H, A)=A$. For any point $x \in X$, the hyperstabilizer of $x$, is defined as $S_{x}=\{h \in H: \phi(h, x)=x\}$. If $S_{x} \neq \emptyset$, then $S_{x}$ is an universal hyperdynamical system $(X, \phi, \mathcal{H})$ of type $L$.

\section{Immersed universal hyperalgebra}

Throughout this paragraph, we work in the $C^{\infty}$ category.

Definition 6.1. An $n$-ary hypergroupoid $\left(H, \lambda_{n}\right)$ is called an immersed universal algebra if $H$ is a smooth real manifold and for all $x_{1}^{n} \in H, f\left(x_{1}^{n}\right)$ is an immersed submanifold of $H$.

Example 6.2. A $p$-plane (with $p \leq n$ ) in $\mathbb{R}^{n}$ is a $p$-dimensional vector subspace of $\mathbb{R}^{n}$ and so it is determined by an ordered set of $p$ independent vectors of $\mathbb{R}^{N}$. We can define a $C^{\infty}$ structure on the set $G\left(p, \mathbb{R}^{n}\right)$ of all $p$-planes in $\mathbb{R}^{n}$, which is called a Grassmann manifold. If $\pi_{1}, \ldots, \pi_{n} \in G\left(p, \mathbb{R}^{n}\right)$, then we define an $n$-ary hyperoperation as follows:

$$
\lambda_{n}\left(\pi_{1}, \ldots, \pi_{n}\right)=\left\{\pi \in G\left(p, \mathbb{R}^{n}\right) \mid \pi \text { is a } p \text {-plane of } \pi_{1}+\cdots+\pi_{n}\right\} .
$$

$G\left(p, \mathbb{R}^{n}\right)$ with this $n$-ary hyperoperation and the $C^{\infty}$ structure of Grassmann manifold is an immersed universal hyperalgebra.

Proposition 6.3. Let $\left(H_{1}, \lambda_{n}\right)$ and $\left(H_{2}, \lambda_{m}\right)$ be immersed universal hyperalgebras. Then $\left(H_{1} \times H_{2}, \lambda_{n} \times \lambda_{m}\right)$ is an immersed universal algebra.

Proof. We know that $H=H_{1} \times H_{2}$ is a smooth manifold. Only, we show that for given $x=\left(x_{1}, \ldots, x_{n}\right) \in H_{1}^{n}, y=\left(y_{1}, \ldots, y_{n}\right) \in H_{2}^{m}$, the set $\lambda_{n}\left(x_{1}, \ldots, x_{n}\right)$ $\times \lambda_{m}\left(y_{1}, \ldots, y_{m}\right)$ is an immersed submanifold of $H$. Since $i_{1}: \lambda_{n}\left(x_{1}, \ldots, x_{n}\right) \rightarrow$ $H_{1}$ and $i_{2}: \lambda_{m}\left(y_{1}, \ldots, y_{n}\right) \rightarrow H_{2}$ are immersions, the map $i: \lambda_{n}\left(x_{1}, \ldots, x_{n}\right) \times$ $\lambda_{m}\left(y_{1}, \ldots, y_{m}\right) \rightarrow H_{1} \times H_{2}$ defined by $i\left(c_{1}, c_{2}\right)=\left(i_{1}\left(c_{1}\right), i_{2}\left(c_{2}\right)\right)$ is an immersion as well. Because, it is a one to one map and $d i\left(c_{1} \times c_{2}\right)=d i_{1}\left(c_{1}\right) \times d i_{2}\left(c_{2}\right)$, which is a one to one map. 
Proposition 6.4. If $\left(H, \lambda_{n}\right)$ is an immersed universal hyperalgebra, then any open subset $U$ of $H$ with the $n$-ary hyperoperation $\lambda_{n}^{U}$ on $U^{n}$ defined by $\lambda_{n}^{U}\left(u_{1}\right.$, $\left.\ldots, u_{n}\right)=\lambda_{n}\left(u_{1}, \ldots, u_{n}\right) \cap U$ is an immersed universal hyperalgebra.

Proof. $U$ with the atlas $A_{U}=\{$ smooth charts $(v, \theta)$ of $H: V \subset U\}$ is a manifold. Moreover, it is a universal hyperalgebra. If $u_{1}, \ldots, u_{n} \in U$, then $i$ : $\lambda_{n}\left(u_{1}, \ldots, u_{n}\right) \rightarrow H$ is an immersion. Hence $i_{U}: \lambda_{n}\left(u_{1}, \ldots, u_{n}\right) \cap U \rightarrow U$ is also an immersion. Therefore $\left(U, \lambda_{n}^{U}\right)$ is an immersed universal hyperalgebra.

Proposition 6.5. If $\left(H, \lambda_{n}\right)$ is an immersed universal algebra and $\psi: H \rightarrow \mathbb{R}^{k}$ is a smooth one to one immersion, then $\psi(H)$ with the $n$-ary hyperoperation

$$
\lambda_{n}^{\psi}\left(\psi\left(h_{1}\right), \ldots, \psi\left(h_{n}\right)\right)=\psi\left(\lambda_{n}\left(h_{1}, \ldots, h_{n}\right)\right)
$$

is an immersed universal hyperalgebra, where the smooth structure of $\psi(H)$ is the smooth structure induced by $\psi$

Proof. It is straightforward.

\section{References}

[1] R. Ameri, Topological (transposition) hypergroups, Ital. J. Pure Appl. Math. No. 13 (2003), 171-176.

[2] R. Ameri and M. M. Zahedi, Hyperalgebraic systems, Ital. J. Pure Appl. Math. No. 6 (1999), 21-32.

[3] P. Corsini, Prolegomena of Hypergroup Theory, Supplement to Riv. Mat. Pura Appl. Aviani Editore, Tricesimo, 1993.

[4] P. Corsini and V. Leoreanu, Applications of Hyperstructures Theory, Kluwer Academic Publishers, Dordrecht, 2003.

[5] G. Crombez and G. Six, On topological n-groups, Abh. Math. Sem. Univ. Hamburg 41 (1974), 115-124.

[6] B. Davvaz, On connection between uncertainty algebraic hypersystems and probability spaces, Internat. J. Uncertain. Fuzziness Knowledge-Based Systems 13 (2005), no. 3, 337-345.

[7] B. Davvaz and T. Vougiouklis, n-ary hypergroups, Iran. J. Sci. Technol. Trans. A Sci. 30 (2006), no. 2, 165-174.

[8] Š. Hošková, Topological hypergroupoids, submitted.

[9] Š. Hošková, J. Chvalina, and P. Račková, Transposition hypergroups of Fredholm integral operators and related hyperstructures (Part I), Journal of Basic Science, 4 (2008), no. $1,43-54$.

[10] Š. Hošková and J. Chvalina, Discrete transformation hypergroups and transformation hypergroups with phase tolerance space, Discrete Math. 308 (2008), no. 18, 4133-4143.

[11] A. Ilookashooly and M. R. Molaei, Immersed hypergroups, Differ. Geom. Dyn. Syst. 10 (2008), 159-162.

[12] V. Leoreanu-Fotea and B. Davvaz, n-hypergroups and binary relations, European J. Combin. 29 (2008), no. 5, 1207-1218.

[13] _ Join n-spaces and lattices, Journal of Multiple Valued Logic and Soft Computing, v.15, 2008 .

[14] _ Roughness in n-ary hypergroups, Inform. Sci. 178 (2008), no. 21, 4114-4124.

[15] M. R. Molaei, Generalized dynamical systems, Pure Math. Appl. 14 (2003), no. 1-2, $117-120$.

[16] R. B. Nelsen, An Introduction to Copulas, 2nd edition, Springer, New York, 2006. 
[17] C. Pelea and I. Purdea, Multialgebras, universal algebras and identities, J. Aust. Math. Soc. 81 (2006), no. 1, 121-139.

[18] M. Sklar, Fonctions de répartition à $n$ dimensions et leurs marges, Publ. Inst. Statist. Univ. Paris 8 (1959), 229-231.

[19] L. Vietoris, Bereiche zweiter Ordnung, Monatsh. Math. Phys. 32 (1922), no. 1, 258-280.

[20] T. Vougiouklis, Hyperstructures and Their Representations, Hadronic Press Monographs in Mathematics. Hadronic Press, Inc., Palm Harbor, FL, 1994.

A. Dehghan NeZhad

Department of Mathematics

YAZD UNIVERSITY

YAZD, IRAN

E-mail address: anezhad@yazduni.ac.ir

B. DAVVAZ

Department of Mathematics

YAZD UNIVERSITY

YAZD, IRAN

E-mail address: davvaz@yazduni.ac.ir 